\title{
CORRESPONDENCE
}

\section{Agronomy and plant breeding are key to combating food crisis}

$\mathrm{SIR}$ - In your Editorial 'A research menu' (Nature 453, 1-2; 2008), you highlight the need to spend more on agricultural science to overcome today's food crisis. But this is not just a matter of greater expenditure - the way in which the money is spent is also important.

Reduced public spending on agricultural research might well be partly to blame for the present crisis. But it is also true that what funding there is has increasingly been directed towards molecular aspects of plant growth and development, arguably at the expense of practical agronomy and breeding issues.

Progress in understanding plant molecular biology is impressive, and useful applications are evident when the trait in question is relatively simple. But improvements in yield and input efficiency - essential for sustainability - stem almost exclusively from traditional breeding and agronomy.

Molecular tools such as gene-expression and metabolite profiling are a long way from becoming incorporated into selection procedures for complextrait breeding. There are many well-characterized quantitative trait loci (stretches of DNA closely linked to the genes that underlie a trait) that affect yield, but there are no clear examples of such loci being successfully backcrossed into highperformance crops.

Diverting most of the limited agricultural-research resources into molecular biology makes it harder to obtain routine funding to improve traditional breeding and agronomy. So, although we agree that a marked increase in funding is necessary, funding bodies should be aware that preferred allocation to molecular biology risks delaying progress on the pressing issue of improving crop productivity.
In this context, European reluctance to fund research into yield improvement has important implications for European as well as developing countries. As the 'green revolution' example shows, such knowledge is useful beyond geographical frontiers.

\section{Lucas Borrás Departamento de Producción Vegetal, Facultad de Ciencias Agrarias, Universidad Nacional de Rosario, S2125ZAA Zavalla, Santa Fe, Argentina Gustavo A. Slafer ICREA, Catalonian Institution for Research and Advanced Studies and Department of Crop and Forest Sciences, University of Lleida, Centre UdL-IRTA, Av. Rovira Roure 191, 25198 Lleida, Spain \\ The ethical basis of the null hypothesis}

SIR — Further to T. Häusler's 'In Retrospect' review of Sinclair Lewis's 1925 novel Arrowsmith (Nature 453, 38; 2008), the book was required reading for graduate courses in professional practice and ethics in the biological sciences that I taught in the 1990s. Arrowsmith's ethical dilemma was whether he should deny some villagers his phage therapy so that they could serve as controls. His conundrum endures to this day - the choice between bequeathing knowledge from a properly designed controlled experiment and risking the health of members of the control group by withholding potentially beneficial treatment.

The control group provides an unbiased test of the null hypothesis, which predicts what to expect if our ideas of how nature works are wrong. It could be argued that it is therefore an ethical obligation for the scientist to take the null hypothesis seriously. No other professional is ethically obliged to consider what might happen if he or she is wrong.

Arrowsmith's employers put the entire ethical burden of choosing the control group and implementing the experiment onto his shoulders. Ethical burdens, however, are properly borne by the entire community. Current practices of having institutional review boards to oversee experiments, obtaining consent from the treated patient, double-blind procedures and full disclosure combine to ensure that the ethical burden of research is shared by all the people involved in the work and is not unfairly placed on a single individual.

John Pastor Department of Biology, University of Minnesota-Duluth, Duluth, Minnesota 55812, USA

\section{Stem-cell urological treatment was not carried out illegally}

SIR - I want to express my displeasure at the News story 'Doctors accused of doing illegal stem-cell trials' (Nature 453, 6-7; 2008).

A prospective cohort study and a prospective randomized trial of our procedure for treating urinary incontinence with autologous myoblasts and fibroblasts were filed with the ethics committee of the University of Innsbruck in February 2001. I cannot explain why these documents are no longer intact in the committee's records. Results of the prospective randomized trial, involving 63 women, were published last year $(\mathrm{H}$. Strasser et al. The Lancet 369, 2179-2186; 2007).

The ethics committee itself emphasized that approval for the study should be applied for at the Arzneimittelbeirat (pharmaceutical committee) of the Austrian Ministry of Health, owing to the novelty of the projects. After receiving the protocols, the Arzneimittelbeirat had no objections to performing clinical studies in June 2002. Andreas Scheil's assertion that the last time approval for studies was given by the government, and not by an ethical committee, was during the Third Reich is insulting.

After our publication in The
Lancet, the ethics committee asked the director of the Ministry of Health's office for public health, Hubert Hrabcik, to reinvestigate the application and approval for the trial published in The Lancet. In October 2007, Hrabcik informed the ethics committee that the Arzneimittelbeirat dealt with the trial published in The Lancet and confirmed that approval by the Arzneimittelbeirat represents ethical approval.

Because of results showing advantages compared with other therapies, and as production of the stem cells required for the treatment of urinary incontinence had been approved by the Ministry of Health, the department of urology decided to offer this therapy outside clinical trials to selected patients who had signed an informed consent. The patient mentioned in your article, Dieter Bollmann, had no postoperative side-effects or complications. I want to emphasize that, although your article states that "patients" are taking legal action, I only know of this single case of a patient suing the hospital.

On 8 May, TILAK (the company that manages the Innsbruck University hospital) and the department of urology of the Medical University of Innsbruck issued a joint statement in which they expressed regret for past misunderstandings, differences of opinion, problems in coordination of procedures, and irritations. A new, extensive clinical study will be performed in close coordination with the ethics committee in reaction to the new European Union directive on advanced therapies.

After the completion of successful clinical studies, it is planned to offer the injection of autologous myoblasts and fibroblasts as standard therapy in the future, to the benefit of the Tyrolean population.

Hannes Strasser Universitätsklinik für Urologie, Medizinische Universität Innsbruck, Anichstrasse 35, 6020 Innsbruck, Austria 